\title{
Pharmacological Approaches to Antitrypanosomal Chemotherapy
}

\author{
Simon L Croft \\ Department of Infectious and Tropical Diseases, London School of Hygiene and Tropical Medicine, Keppel \\ Street, London WC1E 7HT, UK
}

\begin{abstract}
There is an urgent need for new drugs for the chemotherapy of human African trypanosomiasis, Chagas disease and leishmaniasis. Progress has been made in the identification and characterization of novel drug targets for rational chemotherapy and inhibitors of trypanosomatid glycosomal enzymes, trypanothione reductase, ornithine decarboxylase, S-adenosylmethionine decarboxylase, cysteine proteases and of the purine and sterol biosynthetic pathways. However, less attention has been paid to the pharmacological aspects of drug design or to the use of drug delivery systems in the chemotherapy of African trypanosomiasis and Chagas disease. A review of research on pharmacology and drug delivery systems shows that there are new opportunities for improving the chemotherapy of these diseases.
\end{abstract}

Key words: Trypanosoma brucei - Trypanosoma cruzi - drug delivery systems - liposomes - blood brain barrier

Arsenical and antimonial drugs were amongst the first synthetic drugs to be introduced for infectious diseases in the first two decades of this century. It is remarkable that organic derivatives of these heavy metals remain the recommended drugs for the treatment of, respectively, human African trypanosomiasis (sleeping sickness or HAT) and leishmaniasis. No drugs became available for the treatment of Chagas disease until the 1970's when two nitroheterocyclic drugs, nifurtimox and benznidazole, were introduced. All the drugs recommended for the treatment of the trypanosomiases and the leishmaniases have limitations, including either variable efficacy, toxicity, requirements for long courses of parenteral administration, resistance and problems of supply, or a combination of these factors. Although there are several drugs on trial for the chemotherapy of human leishmaniasis many are new formulations of old drugs. There are few drugs of promise for either HAT or Chagas disease and the situation is precarious (De Castro 1993, Wang 1995, Croft et al. 1997).

A major focus for the discovery of novel antitrypanosomal and antileishmanial drugs during the past two decades has been the identification and characterization of biochemical and mo-

\footnotetext{
This work received financial support from the UNDP/ World Bank/WHO Special Programme for Research and Training in Tropical Diseases (TDR).

Fax: +44-171-636.8739. E-mail: s.croft@1shtm.ac.uk Received 12 November 1998

Accepted 19 January 1999
}

lecular targets. Rational approaches to chemotherapy have investigated several targets in detail including the sterol biosynthetic pathway in Trypanosoma cruzi, ornithine decarboxylase in the $T$. brucei complex, trypanothione reductase in Trypanosoma and Leishmania spp., nucleoside phosphotransferases in Trypanosoma and Leishmania spp., glycosomal enzymes in the T. brucei complex, cysteine proteases in T. cruzi and Leishmania, folate metabolism and DNA topoisomerases (see Hunter 1997, Wang 1997 and other references in Coombs \& Croft 1997). Several targets have been characterised structurally and the design of specific inhibitors is well underway .

In contrast the pharmacological aspects of drug design that include aspects to improve drug uptake, activation by pathogen or host cells, absorption and distribution in the host (Gumbleton \& Sneader 1994, Navia \& Chatuverdi 1996) have received less attention. Most reported research in this area have focused on the chemotherapy of leishmaniasis and not the trypanosomiases, including studies on quantitative structure activity relationships to improve lipophilicity and activity (Booth et al. 1987), the lysosomotropic properties of amino acid and dipeptide esters and bis (benzyl)polyamine analogues to enhance accumulation by the Leishmania infected phagosome (Rabinovitch 1989, Baumann et al. 1991), and carrier molecules to aid targetting of drug to parasite or parasite host cell, for example allopurinolglycosylated polymers to receptors on Leishmania infected macrophages (Negre et al. 1992). Drug delivery systems (cellular, particulate and vesicular) designed to enhance drug targeting to infected tissues, reduce metabolism by the host or toxicity 
to the host, or enable sustained release of drug within the host have also been explored for the treatment of leishmaniasis. The most successful application of drug delivery systems has certainly been the use of lipid amphotericin B formulations for visceral leishmaniasis both in experimental models (Croft et al. 1991, Berman et al. 1992) and clinical trials (Berman et al. 1998); liposomes have also proved effective in experimental cutaneous leishmaniasis (Yardley \& Croft 1997). Alternatively, nanoparticles incorporating primaquine and pentamidine have also shown to be useful drug carriers in experimental visceral leishmaniasis (Rodrigues et al. 1994). So if these approaches can benefit the chemotherapy of leishmaniasis, why not also the chemotherapy of the trypanosomiases?

\section{HUMAN AFRICAN TRYPANOSOMIASIS}

In the first stages of HAT parasites are restricted to the haemolymphatic system and drugs, namely pentamidine and suramin are available for the treatment of early stage disease. The major problem in treatment of HAT is the second stage, characterized by the presence of trypanosomes in the Central Nervous System (CNF) and the cerebrospinal fluid (CSF). A re-appraisal of the pharmacokinetics of the only available drug for the treatment of both $T$. b. gambiense or T. b. rhodesiense second stage disease, the trivalent arsenical melarsoprol $\left[\mathrm{Mel} \mathrm{B}^{\hat{a}}\right]$, may help to improve treatment. Melarsoprol, with a masked reactive arsenoxide group, is lipid soluble and should gain easy access to the brain, although suprisingly low levels of drug have been detected in the CNS, only 0.5 to $5 \%$ of plasma levels (Pepin \& Milord 1994). Recommended treatment schedules are of three or four courses spaced by an interval of at least one week. It has been suggested that these schedules may not be the most effective and may also be responsible in part for the frequently reported side effects, the most severe being a reactive encephalopathy in up to $10 \%$ of the treated patients with a mortality of up to 5\% (Pepin \& Milord 1994). Bioassay and Elisa techniques (Maes et al. 1988, Burri et al. 1993) have been used to determine drug levels of melarsoprol and active metabolites in serum and CSF and an elimination half life time of $35 \mathrm{hr}$ in the blood (Burri et al. 1993). Since blood levels decreased to zero in between the courses, an alternative treatment protocol consisting of 10 consecutive daily doses has been suggested and a preliminary clinical study in Zaire gave promising results with the 10-day regime (Burri et al. 1995). A full clinical evaluation is now underway. A re-examination of the pharmacokinetics of pentamidine, a highly charged diamidine that should not cross the blood-brain barrier (BBB) and is ineffective against second stage disease, was suprisingly detected at $0.5-0.8 \%$ of plasma levels in the CSF (Bronner et al. 1991). This penetration of the CNS may be due to a breakdown of the blood-brain barrier (BBB) resulting from the inflammatory response that accompanies the CNS infection. Eflornithine, or DL-a-difluoromethylornithine (DFMO), the only new drug to be developed for the treatment of late-stage HAT since the introduction of melarsoprol in 1949, was registered for use in the early 1990s and has been used to treat many T. $b$. gambiense cases including many melarsoprol-refractory cases (Pepin \& Milord 1994). Eflornithine shows excellent penetration into the CNS and high levels have been detected in CSF compared to plasma levels in adults, although lower levels were detected in the CSF of children (Milord et al. 1993). The inactivity of eflornithine against East African sleeping sickness is due to innate tolerance of T. $b$. rhodesiense to the drug (Iten et al. 1995) and not CNS penetration.

The BBB describes the continuous tight junctions between the endothelial cells of the cerebral capillaries that restrict the movement of polar solutes into the brain and are important in controlling the composition of the fluids that bathe the brain. Although fenestrated capillaries in the choroid plexus allow a more direct exchange between blood and brain fluids, this area constitutes only $1 / 5000$ of the total area for exchange of the tight BBB capillaries. This high level of control of access has clear implications for delivery of drugs to the brain. However, the BBB is not an inert barrier; there are special transport systems that enable access of amino acids, glucose and specific macromolecules into the brain (Begley 1996). In addition passive diffusion of low molecular weight lipophilic compounds, for example melarsoprol, is possible. A vervet monkey model has recently been established to evaluate the capability of compounds to cross the BBB (Brun, pers. comm.). Some compounds found to be highly active in vitro or the acute rodent model of infection, for example the nucleoside analogues S-HPMPA (Kaminsky et al. 1996) and the diamidine trybizine hydrochloride (Bacchi, pers. comm., Kaminsky \& Brun, pers. comm.) have not been pursued for further development because of their apparent inability to cross the BBB in this model (Brun, pers. comm.).

A search for new treatments for other CNS diseases, for example Alzheimer's and Parkinson's diseases and viral infections has focused attention on novel approaches to improve delivery of drugs across the BBB. Two such examples for HIV infections of the brain include the design of lipophilic nucleoside derivatives (Ford et al. 1995) and the conjugation of zidothymidine to a lipophilic 
carrier that crosses the BBB to become more hydrophilic and trapped in the brain (Brewster et al. 1997). Peptides, for example somastatin analogues, have also been designed with enhanced permeability (see Begley 1996). There are many paradigms that could be included in the design of new drugs for HAT. One group of drugs that do cross the BBB are novel antiprotozoals, the alkyllysophospholipids (ALPs), that include miltefosine, edelfosine and ilmofosine, active against $T$. brucei bloodstream forms in vitro with $\mathrm{ED}_{50}$ values in the range of 10-40 mM (Croft et al. 1996). Although they do not appear to be effective against acute $T$. brucei infections in mice (Croft \& Rock, unpublished) their potential against chronic CNS infections is being investigated in combination with other antitrypanosomal drugs, a well established strategy for looking for CNS activity (Jennings 1993).

The drug targeting approach towards the treatment of African trypanosomiasis has been tested in model systems, predominantly against $T$. brucei. Williamson et al. (1981) observed that the anticancer drug daunorubicin was highly active against T.brucei in vitro but inactive in vivo. Although initial efforts to improve daunorubicin uptake into bloodstream form trypanosomes by linking the drug to DNA were ineffective, further studies using protein carriers were more successful, possibly through increased uptake by endocytosis and/ or reduced plasma clearance. Daunorubicin was most active when linked to BSA (as opposed to ferritin) by a labile covalent linkage (as opposed to a stable linkage) and $90 \%$ animals were cured (Williamson et al. 1981). This approach was extended using particulate carriers with daunorubicin conjugated to agarose beads or adsorbed to polycyanoacrylate nanoparticles, shown to enter the parasites via the flagellar pocket and reside in lysosomal vesicles. In vivo activity of daunorubicin-nanoparticle formulations was not promising, possibly due to retention of drug within the parasite lysosomes unreleased from the carrier and unable to access the nuclear target sites (Golightly et al. 1988). Unloaded nanoparticles were shown to have some antitrypanosomal activity (Lherm et al. 1987, Golightly et al. 1988). In another approach involving a molecular carrier, the anticancer drug cisplatin was more effective against $T$. congolense in mice when complexed with polyglutamic acid than when administered free, probably due to both a longer plasma half-life and improved uptake (Meshnick et al. 1984). The applicability of these approaches to the treatment of T. brucei infections always appeared to be limited especially in view of the CNS stage of trypanosomiasis. However, recent research on the blood- brain barrier has indicated receptor mediated transcytosis, and there have been attempts to improve drug uptake using a monoclonal antibody OX26 to the BBB transferrin receptor as a drug carrier (Friden et al. 1993). This again opens up an area for trypanosomiasis research. A range of other transporters, for example the glucose carrier GLUT-1 and the system-L transporter of neutral amino acids could also be exploited for drug design and delivery across the BBB (see Begley 1996). Most remarkable are the observations that poly(butyl)cyanoacrylate nanoparticles $(230 \mathrm{~nm}$ diameter) coated with the detergent polysorbate80 appear to penetrate the BBB and release quantities of drug within the brain (Kreuter et al. 1995, Schroder \& Sabel 1996). A re-examination of the nanoparticle delivery approach for experimental CNS trypanosomiasis should be made. Attempts at drug delivery to the brain using liposomes has been unsuccessful (see Begley 1996).

Yet another approach used to assist the delivery of drugs across the BBB to the CNS involves opening intercellular junctions. Osmotic shock following administration of a hyperosmotic solution, for example of mannitol, has been used clinically to increase access of anti-tumour drugs (Neuwelt $\&$ Barnett 1989). An indication of the applicability of this approach to T. brucei infections in rats was demonstrated using hyperosomotic lithium chloride to increase the level of diminazene aceturate in the brain of infected animals and to improve therapy (Odika et al. 1995a,b). A more recent and sophisticated tactic is based upon receptor-mediated permeabilization of tight junctions by bradykinin. A bradykinin agonist, RMP-7, has been shown to open tight junctions of BBB capillaries and enhance drug entry into the brain (Bartus et al. 1996, Elliott et al. 1996). It is strange in this context to mention DFMO again. Ornithine decarboxylase levels in microvasculature increase following BBB breakdown induced by mannitol; DFMO reverses BBB breakdown through inhibition of ornithine decarboxylase (Koenig et al. 1989).

\section{SOUTH AMERICAN TRYPANOSOMIASIS}

The wide tissue and cell distribution of intracellular T. cruzi amastigotes during both the acute and chronic phases makes Chagas disease a more difficult subject for drug targeting in comparison to leishmaniasis where amastigotes are restricted to macrophages and HAT where there is a defined problem of the BBB. Perhaps because of this little attention has been paid to drug delivery and the pharmacokinetics of anti-T. cruzi drugs since the studies of Avila et al. (1979) and Avila (1983). The lysosomotropic properties of a DNA- 
pharmacophore complex, ethidium bromide-DNA were initially studied. A suppressive effect of a $T$. cruzi $\mathrm{Y}$ strain infection of mice was demonstrated but not cure (Avila et al. 1979). One explanation offered for this was that T. cruzi amastigotes survive in host cell cytoplasm whereas the drug was targeted to the phagosomal compartment and was not released. Subsequently, 4-amino-pyrazolopyrimidine (APP), a compound similar in structure to allopurinol which has been on clinical trial for Chagas' disease (Gallerano et al. 1990), was derivatised with glucosamine and conjugated to BSA. BSA-gluAPP together with BSA-leugluAPP and BSA-ala-leu-gluAPP derivatives had little activity against T.cruzi $\mathrm{Y}$ strain acute infections in mice. However, albumin-leu-ala-leugluAPP and albumin-ala-leu-ala-leu-gluAPP were significantly efficacious at $0.1 \mathrm{mg} / \mathrm{kg}$, animals surviving until 250-290 days in comparison to survival times of 13 days for controls and 52 days for free drug treated animals. The high activity of these conjugates parallelled the ability of APP release from carrier molecule by purified lysosmal enzymes (Avila 1983). In a further study formycin $\mathrm{B}$, another purine analogue, and APP were adsorbed onto $200 \mathrm{~nm}$ polyisobutylcyanoacrylate nanoparticles. These nanoparticles also significantly increased the survival time of T.cruzi $\mathrm{Y}$ acutely infected mice in comparison to free drug and unloaded carriers which were relatively ineffective (Avila 1983). Do any drug delivery systems offer potential for the treatment of Chagas disease?

Amphotericin B is highly active against $T$. cruzi (De Castro et al. 1994) as these parasites, like fungi and Leishmania, possess ergosterol as the predominant membrane sterol to which the drug binds in preference to cholesterol. However, the use of amphotericin B in the treatment of mycoses, leishmaniasis and Chagas disease has been limited by a wide range of toxic side effects. Lipid amphotericin B formulations, which overcome the toxic limitations of the parent drug, were developed for the treatment of systemic mycoses and are now also used for the treatment of visceral leishmaniasis. We have shown that they also have activity against established T. cruzi $\mathrm{Y}$ strain infections in mice. The activities of Amphotericin B liposomes (AmBisome $^{\mathrm{O}}$ ), amphotericin B colloidal dispersion $\left(\right.$ Amphocil ${ }^{\grave{O}}$ ) and amphotericin B lipid complex (Abelcet ${ }^{\circ}$ ) (see Hartsel \& Bolard 1996) formulations were compared with free drug in vitro against intracellular amastigotes in Vero cells and macrophages; there was no significant variation of activity against amastigotes in Vero cells but there was variation in macrophages, probably due to differences in accumulation (Yardley \& Croft, unpub- lished). In vivo a single dose of $25 \mathrm{mg} / \mathrm{kg}$ or 12.5 $\mathrm{mg} / \mathrm{kg}$ i.v. increased the survival time of all mice significantly in comparison to controls, free drug at the maximum tolerated dose of $0.5 \mathrm{mg} / \mathrm{kg}$, and a dose of $6.25 \mathrm{mg} / \mathrm{kg}$ (Yardley \& Croft, unpublished). AmBisome ${ }^{O}$ was best tolerated by infected mice; the formulation also has a longer half-life than parent compound (Proffitt et al. 1991). We are currently examining the curative activity of this formulation and its efficacy in a chronic model of infection.

It is noteworthy that since the early publications on benznidazole and nifurtimox in the 1970's there has been little consideration of the pharmacokinetics of anti-T. cruzi drugs. With the continuing interest in the potential of sterol biosynthesis inhibitors for the treatment of Chagas disease (Urbina 1997) some attention should be paid to the pharmacokinetics of these drugs in T.cruzi infected hosts. The variation of distribution and metabolism of azoles has been considered in relation to treatment of fungal infections (Hay 1993) as have the pharmacokinetics of other sterol inhibitors that have been suggested for combination therapy (Urbina 1997), for example HMG-CoA reductase inhibitors (Hamelin \& Turgeon 1998) and terbinafine (Faergemann 1997).

\section{CONCLUSION}

Experience with arsenicals for HAT and antimonials for leishmaniasis has shown how re-examination of pharmacokinetics can lead to new drug regimes and improved therapy. A better understanding of the bioavailability and distribution of drugs and the local host physiology at the site of infection, combined with an understanding of the potential of drug design and drug delivery systems provides new opportunities for improving the chemotherapy of the trypanosomiases.

\section{ACKNOWLEDGEMENTS}

To Reto Brun, Ronald Kaminsky and Vanessa Yardley for comments on the manuscript.

\section{REFERENCES}

Avila JL 1983. New rational approaches to Chagas' disease chemotherapy. Interciencia 8: 405-417.

Avila JL, Bretaña A, Avila A 1979. Chemotherapy with ethidium bromide-DNA complex in established Chagas' disease. Am J TropMed Hyg 28: 456-460.

Baumann RJ, McCann PP, Bitonti AJ 1991. Suppression of Leishmania donovani by oral administration of a bis(benzyl)polyamine analog. Antimicrobial $\mathrm{Ag}$ Chemo 35: 1403-1407.

Bartus RT, Elliott P, Hayward N, Dean R, McEwen EL, Fisher SK 1996. Permeability of the blood brain barrier by the bradykinin agonist, RMP-7: evidence for a sensitive, auto-regulated, receptor-mediated system. Immunopharmacology $33: 270-278$. 
Begley DJ 1996. The blood-brain barrier: principles for targeting peptides and drugs to the central nervous system. J Pharm Pharmacol 48: 136-146.

Berman JD, Badaro R, Thakur CP, Wasunna KM, Bebehani K, Davidson R, Kuzoe F, Pang L, Weerasuriya K, Bryceson ADM 1998. Efficacy and safety of liposomal amphotericin B (AmBisome) for visceral leishmaniasis in endemic developing countries. Bull WHO 76: 25-32,

Berman JD, Ksionski G, Chapman WL, Waits VB, Hanson WL 1992. Activity of amphotericin B cholesterol dispersion (Amphocil) in experimental visceral leishmaniasis. Antimicrobial Ag Chemo 36: 1978-1980.

Booth RG, Selassie CD, Hansch C, Santi DV 1987. Quantitative structure-activity relationship of triazine-antifolate inhibition of Leishmania dihydrofolate reductase and cell growth. J Med Chem 30: 12181224.

Brewster ME, Anderson WR, Webb AI, Pablo LM, Meinsma D, Moreno D, Derendorf H, Bodor N, Pop E. 1997. Evaluation of a brain-targeting zidovudine chemical delivery system in dogs. Antimicrobial Ag Chemo 41 :122-128.

Bronner U, Doua F, Ericsson O, Gustafsson LL, Miezan TW, Rais M, Rombo L. 1991. Pentamidine concentrations in plasma, whole blood and cerebrospinal fluid during treatment of Trypanosoma gambiense infection in Cote d'Ivoire. Trans $R$ Soc Trop Med Hyg 85: 608-611.

Burri C, Baltz T, Giroud C, Doua F, Welker HA, Brun, R. 1993. Pharmacokinetic properties of the trypanocidal drug melarsoprol. Chemotherapy 39: 225-234.

Burri C, Blum J, Brun, R. 1995. Alternative application of melarsoprol for treatment of T. b. gambiense sleeping sickness. Ann Soc Belg Med Trop 75: 65-71.

Coombs GH, Croft SL 1997. Molecular basis of drug design and resistance. Parasitology 114 Suppl.

Croft SL, Davidson RN, Thornton E 1991. Liposomal amphotericin B in the treatment of visceral leishmaniasis. J Antimicrob Chemother 28 Suppl. B: 111-118.

Croft SL, Snowdon D, Yardley V 1996. The activities of four anticancer alkyllysophospholipids against Leishmania donovani, Trypanosoma cruzi and Trypanosoma brucei. J Antimicrob Chemother 38: 10411047.

Croft SL, Urbina JA, Brun R. 1997. Chemotherapy of human leishmaniasis and trypanosomiasis, p. 245257. In G Hide, JC Mottram, GH Coombs, PH Holmes (eds), Trypanosomiasis and Leishmaniasis, CAB International, Wallingford, UK.

De Castro SL 1993. The challenge of Chagas disease chemotherapy: an update of drugs assayed against Trypansoma cruzi. Acta Trop 53: 83-98.

De Castro SL, Soeiro MNC, Higashi KO, Meirelles MNL 1994. Differential effect of amphotericin B on the three evolutive stages of Trypanosoma cruzi and on the host cell-parasite interaction. Braz J Med Biol Res 26: 1219-1229.

Elliott PJ, Hayward NJ, Dean RL, Blunt DG, Bartus RT 1996. Intravenous RMP-7 selectively increases uptake of carboplatin into rat brain tumours. Cancer
Res 56: 3998-4005.

Faergemann J 1997. Pharmacokinetics of terbinafine. Rev Contemp Pharmacotherapy 8: 289-297.

Ford H Jr, Siddiqui MA, Driscoll JS, Marquez VE, Kelley JA, Mitsuya H, Shirasaka T 1995. Lipophilic, acidstable, adenosine deaminase-activated anti-HIV prodrugs for central nervous system delivery. 2. 6halo and 6-alkoxy prodrugs of 2'-beta-fluoro-2', 3'dideoxyinosine. J Med Chem 38: 1189-1195.

Friden PM, Walus LR, Watson P, Doctrow SR, Kozararich JW, Bäkman C, Bergman H, Hoffer B, Bloom F, Granholm A-C 1993. Blood-brain barrier penetration and in vivo activity of an NGF conjugate. Science 259: 373-377.

Gallerano RH, Marr JJ, Sosa JJ 1990. Therapeutic efficacy of allopurinol in patients with chronic Chagas' disease. Am J Trop Med Hyg 43: 159-166.

Golightly L, Brown JE, Mitchell JB, Brown JR 1988. Trypanocidal activity of free and carrier bound daunorubicin. Cell Biol Int Rep 12: 77-83.

Gumbleton M, Sneader W 1994. Pharmacokinetic considerations in rational drug design. Clin Pharmacokinet 26: 161-168.

Hamelin BA, Turgeon J 1998. Hydrophilicity/ lipophilicity: relevance for the pharmacology and clinical effects of HMG-CoA reductase inhibitors. Trends Pharmacol Sci 19: 26-37.

Hartsel S, Bolard J 1996. Amphotericin B: new life for an old drug. Trends Pharmacol Sci 17: 445-449.

Hay RJ 1993. The need for new antifungals - a clinical appraisal, p. 163-181. In PH Bentley, R Ponsford, (eds), Recent Advances in the Chemistry of Anti-infective Agents, Royal Society of Chemistry.

Hunter WN 1997. A structure-based approach to drug discovery; crystallography and implications for the development of antiparasite drugs. Parasitology 114 Suppl: S17-S29.

Iten M, Matovu E, Brun R, Kaminsky R 1995. Innate lack of susceptibility of Ugandan Trypanosoma brucei rhodesiense to DL-a-difluoromethylornithine (DFMO). Trop Med Parasitol 46: 190-195.

Jennings FW 1993. Combination chemotherapy of CNS trypanosomiasis. Acta Trop 54: 205-213.

Kaminsky R, Schmid C, Grether Y, Holy A, De Clercq E, Naesens L, Brun R 1996. (S)-9-(3-hydroxy-2phosphonylmethoxypropyl)adenine [(S)-HPMPA]: a purine analogue with trypanocidal activity in vitro and in vivo. Trop Med Intern Hlth 1: 255-263.

Koenig H, Goldstone AD, Lu CY 1989. Polyamines mediate the reversible opening of the blood-brain barrier by the intracarotid infusion of hyperosmolal mannitol. Brain Res 483: 110-116.

Kreuter J, Alyautdin RN, Kharkevich DA, Ivanov AA 1995. Passage of peptides through the blood-brain barrier with colloidal particles (nanoparticles). Brain Res 674: 171-174.

Lherm C, Couvreur P, Loiseau P, Bories C, Gayral P 1987. Unloaded polyisobutylcyanoacrylate nanoparticles: efficiency against bloodstream trypanosomes. J Pharm Pharmacol 39: 650-652.

Maes L, Vanderveken M, Hamers R, Doua F, Cattand P 1988. The monitoring of trypanocidal treatment with 
a sensitive ELISA method for measuring melarosprol levels in serum and in cerborspinal fluids. Ann Soc Belg Med Trop 68: 219-231.

Meshnick SR, Brown SD, Smith G 1984. Capacity of a cis-diammineplatinum[II] - polyglutamic acid complex to cure Trypanosoma congolense infections in mice. Antimicrobial Ag Chemo 25: 286-288.

Milord F, Loko L, Ethier L, Mpia B, Pepin J 1993. Eflornithine concentrations in serum and cerebrospinal fluid of 63 patients treated for Trypanosoma brucei gambiense sleeping sickness. Trans $R$ Soc Trop Med Hyg 87: 473-477.

Navia MA, Chaturvedi PR 1996. Design principles for orally bioavailable drugs. Drug Discovery Today 1: 179-189.

Negre E, Chance ML, Hanboula SY, Monsigny M, Roche A-C, Mayer RM, Hommel M 1992. Antileishmanial drug targeting through glycolsylated polymers specifically internalized by macrophage membrane lectins. Antimicrobial Ag Chemo 36: 2228-2232.

Neuwelt EA, Barnett PA 1989. Blood-brain barrier disruption in th treatment of brain tumours, p. 107-193. In EA Neuwelt, Implications of the Blood-brain Barrier and its Manipulation, vol. 2, Clinical Aspects, Plenum Press, New York.

Odika IE, Asuzu IU, Anika SM 1995a. The effects of hyperosmolar agents lithium chloride and sucrose on the brain concentration of diminazene aceturate in rats. Acta Trop 60: 119-125.

Odika IE, Asuzu IU, Anika SM 1995b. The chemotherapeutic efficacy of diminazene aceturate and lithium chloride against relapse infection of Trypanosoma brucei brucei in rats. Trop Med Parasitol 46: 99-
102.

Pepin J, Milord F 1994. The treatment of Human African Trypanosomiasis. Adv Parasitol 33: 1-47.

Proffitt RT, Satorius A, Chiang SM, Sullivan L, AdlerMoore J 1991. Pharmacology and toxicology of a liposomal formulation of amphotericin B (AmBisome) in rodents. J Antimicrob Chemother 28 Suppl. B: 49-61.

Rabinovitch M 1989. Leishmanicidal activity of amino acid and peptide esters. Parasitol Today 5: 299-301.

Rodrigues Jr. JM, Croft SL, Fessi H, Bories C, Devissaguet J-P 1994. The activity and ultrastructural localization of primaquine-loaded poly $(\mathrm{d}, 1-$ lactide) nanoparticles in Leishmania donovani mice. Trop Med Parasitol 45: 223-228.

Schroder U, Sabel BA 1996. Nanoparticles, a drug carrier system to pass the blood-brain barrier, permit central analgesic effects of i.v. dalargin injections. Brain Res 710: 121-124.

Urbina JA 1997. Lipid biosynthesis pathways as chemotherapeutic targets in kinetoplastid parasites. Parasitology 114 Suppl.: S91-S99.

Wang CC 1995. Molecular mechanisms and therapeutic appraoches to the treatment of African trypanosomiasis. Annu Rev Pharmacol Toxicol 35: 93-127.

Wang CC 1997. Validating targets for antiparasite chemotherapy. Parasitology 114 Suppl.: S31-S44.

Williamson J, Scott-Finnigan TJ, Hardman MA, Brown JR 1981. Trypanocidal activity of daunorubicin and related compounds. Nature 292: 466-467.

Yardley V, Croft SL 1997. Activity of liposomal amphotericin B against experimental cutaneous leishmaniasis. Antimicrobial Ag Chemo 41: 752-756. 